\title{
Detection of Early Stage Precursor during Formation of Plastic Crystal Ethanol from the Supercooled Liquid State: A Simultaneous Dielectric Spectroscopy with Neutron Diffraction Study
}

\author{
Alejandro Sanz, ${ }^{1, *}$ Aurora Nogales, ${ }^{1}$ Inés Puente-Orench, ${ }^{2,3}$ Mónica Jiménez-Ruiz, ${ }^{2}$ and Tiberio A. Ezquerra ${ }^{1}$ \\ ${ }^{1}$ Instituto de Estructura de la Materia, IEM-CSIC, Serrano 121, 28006 Madrid, Spain \\ ${ }^{2}$ Institut Laue-Langevin, BP 156-38042 Grenoble Cedex 9, France \\ ${ }^{3}$ Instituto de Ciencia de Materiales de Aragón, ICMA-CSIC, Pedro Cerbuna 12, 50009 Zaragoza, Spain
}

(Received 25 March 2011; published 6 July 2011)

\begin{abstract}
Transformation of deuterated ethanol from supercooled liquid into a plastic crystal or rotator phase is investigated by means of a particular experimental setup combining simultaneously dielectric spectroscopy with neutron diffraction techniques. We demonstrate that, previous to the growth of the bcc lattice of the plastic crystal phase, the formation of a precursor or intermediate phase through a liquid-liquid phase separation takes place. Once this precursor phase is formed, subsequent (plastic) crystalline nucleation and growth is expected to develop.
\end{abstract}

DOI: 10.1103/PhysRevLett.107.025502

PACS numbers: 61.05.F-, 64.70.kt, 77.22.Gm

Plenty of natural and daily situations are closely linked to phase transitions in matter. From an energetic point of view, the phase transition proceeds when the counterbalance between the internal energy $(U)$ and the entropy $(S)$ minimizes the free energy $F=U-T S$ [1-3]. The liquidsolid or freezing transitions are referred to as first-order phase transitions where the quantities derived from the free energy discontinuously change. In such first-order phase transformations, the two phases must have a distinct density, state of order, and free energy at the corresponding temperature and pressure. Therefore, these are commonly classified as energy-driven phase transitions. Nevertheless, other freezing processes can take place via a partial ordering of the molecules where the density during the phase transition slightly changes. The latter is then understood to be produced by some molecular rearrangements allowing the stabilization of the new phase due to an increase in entropy instead of through a minimization of the internal energy [4]. The transformation of supercooled liquids into rotational disordered crystals is a well-known example of these entropy-driven transitions. In this case, an increase of the rotational mobility (increase of rotational entropy) provides the necessary stabilization of the growing phase [4-8]. It has also been pointed out that these small molecular rearrangements, characteristic of the entropydriven phase transitions, typically take place within the deep undercooled and highly heterogeneous dynamic regime close to the structural glass-transition temperature $T_{g}$ $[9,10]$. In the special case of ethanol, both energy and entropy-driven phase transitions are detected in the supercooled regime depending on the temperature window.

Below the melting temperature $T_{m}$ and depending on the thermal history, ethanol exists either as a supercooled liquid (SCL) (translational and rotational disorder), monoclinic crystalline solid (C) (translational and rotational order), or plastic crystal (PC) (translational order but rotational disorder). The transformation of ethanol from the SCL state into a rotational disordered PC was postulated a long time ago by Haida et al. [11] thanks to calorimetric measurements in the temperature range between the $T_{g}$ and the $T_{m}$. They detected a metastable crystalline phase with a small entropy of fusion, allowing the authors to speculate that this phase belonged to the PC category. Since this pioneering work, several papers have appeared that are related to the polymorphism of ethanol [12-15]. In addition, ethanol is considered a paradigmatic glass former, extensively studied in the glassy and supercooled regimes. Most of these works have been devoted to the observation of how the state of order of supercooled ethanol affects its dynamics above and below the $T_{g}$ $[5,9,16-19]$. It has been found that the positional order of the ethanol molecules modifies the dynamics in a different manner depending on the time scale explored. While the microscopic dynamics in the ps regime as explored by quasielastic neutron scattering is enhanced in the PC phase in comparison to the SCL, the dynamics in the SCL is faster at macroscopic-mesoscopic scales as indicated by dielectric spectroscopy (DS) measurements. This larger rotational mobility at the ps time scale has been considered to play a crucial role on the thermodynamic stabilization of the plastic crystal phase with respect to the supercooled liquid $[4,19]$. Another interesting point is the more fragile character of the SCL than that of the PC. These differences have been rationalized in terms of the extent of the translational motions (frozen for the PC) and the strength of the hydrogen bonds $[5,16,19]$. It has also been demonstrated that in both SCL and PC ethanol the rotations dominate the structural relaxation processes [16].

In this Letter, we directly correlate the dynamic and structural changes occurring in SCL ethanol during ordering into the PC phase. We perform a simultaneous monitorization, in real time, of both the crystal development 
and the dynamic alterations in the amorphous phase. We accomplish this task, by coupling neutron diffraction (ND) and dielectric spectroscopy techniques, providing a complete picture of the ordering process of ethanol into the PC phase [20]. DS has been widely used to study the molecular relaxation of monohydroxy alcohols, especially for elucidating the microscopic origin of the main Debye process observed at low frequencies. This strong Debye peak does not possess the main features of the typical structural or $\alpha$ relaxation, and different models have been proposed in order to explain the origin of this Debye relaxation, but they are always connected to the hydrogen-bonding network dynamics [21-23]. Following the experimental details described in Ref. [24], we have quenched (cooling $>6 \mathrm{~K} / \mathrm{min})$ liquid deuterated ethanol $\left(\mathrm{CD}_{3} \mathrm{CD}_{2} \mathrm{OD}\right)$ into the glassy state (structural glass) from room temperature and heated it up above the $T_{g}$ afterwards (SCL). If the SCL ethanol is annealed between 105 and $110 \mathrm{~K}$, the PC form is generated. Finally, $\mathrm{PC}$ ethanol tends to crystallize into the monoclinic phase at temperatures above $115 \mathrm{~K}$. The simultaneous ND and DS experiments have been carried out at the D1B diffractometer at ILL (Grenoble, France) at a fixed wavelength of $\lambda=2.52 \AA$. A detailed description of the ND-DS setup can be found elsewhere [20,25]. The transformation of SCL ethanol into the PC phase was studied at a fixed temperature of $105 \mathrm{~K}$. Selected ND patterns and DS curves, recorded simultaneously as a function of time, are collected in Fig. 1. The disordered state of the starting sample is confirmed by the typical amorphous halo [see Fig. 1(b)] observed for the initial frames. As the phase transition advances, the characteristic reflections of the bcc lattice of the PC phase superimpose to the amorphous halo. In parallel, as shown in Fig. 1(a) the main dielectric relaxation peak of SCL ethanol becomes broader and shifts to lower frequencies during the phase transition. Deuterated ethanol, when totally transformed into the PC or rotator phase, exhibits a new dielectric process at lower frequencies and is considered a fingerprint of its structural relaxation. Unlike it was reported in a previous work [5], it is worth mentioning that our dielectric loss data do not exhibit an isosbestic frequency between the two main coexisting relaxations. In principle, this finding suggests that our experimental results cannot be interpreted by a simple two-phase model. In order to elucidate unequivocally the structure and the fraction of the sample transformed into the $\mathrm{PC}$ phase, here we consider the diffraction patterns as a linear combination of the ordered $\left(N_{\mathrm{PC}}\right)$ and amorphous $\left(N_{\mathrm{SCL}}\right)$ contributions as follows: $I(Q, t)=$ $N_{\mathrm{PC}}(t) I_{\mathrm{PC}}(Q)+\left[1-N_{\mathrm{PC}}(t)\right] I_{\mathrm{SCL}}(Q)$, where $I_{\mathrm{PC}}$ is the intensity from the Bragg peaks and $I_{\mathrm{SCL}}$ is the intensity from the amorphous halo. A detailed description of the procedure to estimate the weighting factor for each phase can be found elsewhere $[25,26]$. As an example of the fitting procedure of the neutron diffraction patterns, in Fig. 2 we show the best fit of the experimental data for an intermediate time of $4.8 \mathrm{~h}$ to the linear combination depicted above,
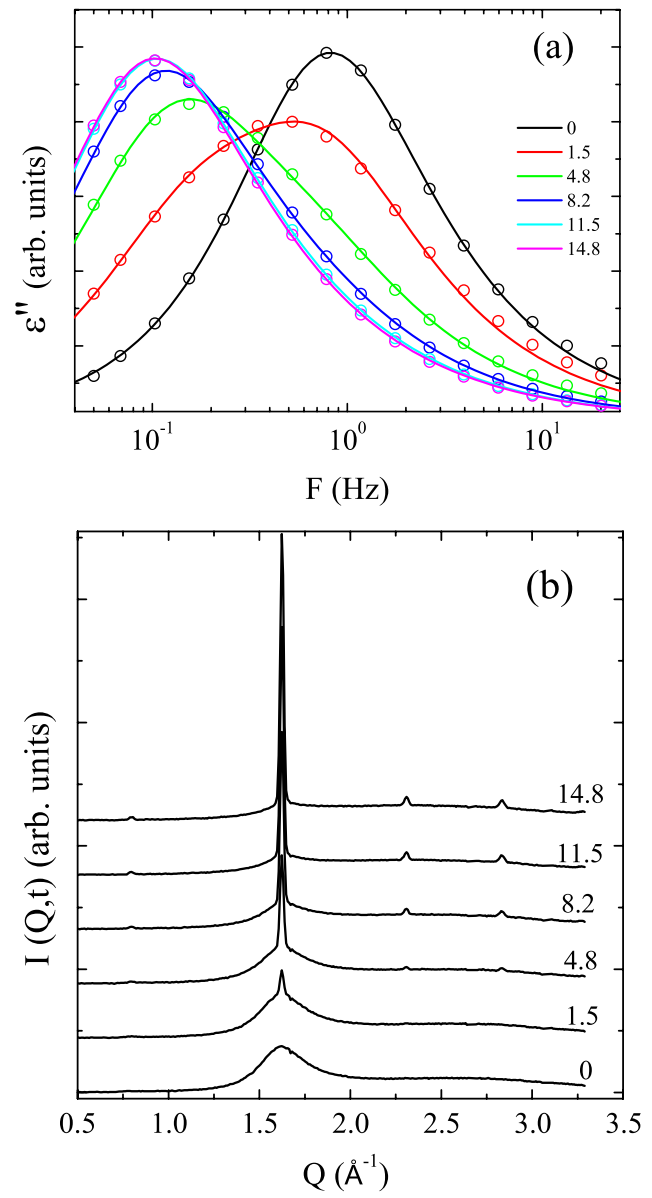

FIG. 1 (color online). Selected snapshots during isothermal transformation of ethanol into PC phase. Simultaneous dielectric loss spectroscopy curves (a) and neutron diffraction patterns (b) are presented during isothermal annealing at $105 \mathrm{~K}$. ND patterns for every time are represented as a function of the scattering vector $\mathbf{Q}$. DS spectra are given for every time as a function of the frequency. Every pattern was recorded with an acquisition time of $10 \mathrm{~min}$. Annealing time is indicated in hours.

with the corresponding contribution from the SCL and PC fractions. Resulting from the fitting analysis, the inset of Fig. 2 displays the fraction of the PC phase $\left(N_{\mathrm{PC}}\right)$ as a function of time at $105 \mathrm{~K}$. These results indicate the full transformation of the SCL into the PC phase after approximately $14 \mathrm{~h}$. In a first approach, we attempted to describe the dielectric signal as an addition of two Cole-Davidson (CD) functions, associated with each of the coexisting mobile phases. The Cole-Davidson function is a particular case of the general Havriliak-Negami (HN) equation with the symmetric broadening parameter $b$ equal to 1 . The $\mathrm{HN}$ fitting function is expressed as

$$
\epsilon^{\prime \prime}(\omega)=\operatorname{Im}\left[\epsilon^{*}(\omega)\right]=\operatorname{Im}\left[\epsilon_{\infty}+\Delta \epsilon\left(1+\left(i \omega \tau_{\mathrm{HN}}\right)^{b}\right)^{-c}\right],
$$

where $\tau_{\mathrm{HN}}$ is the characteristic relaxation time, $c$ is the asymmetric broadening parameter, and $\Delta \epsilon$ is the dielectric strength, the latter being directly related to the fraction of mobile particles contributing to the relaxation mode 


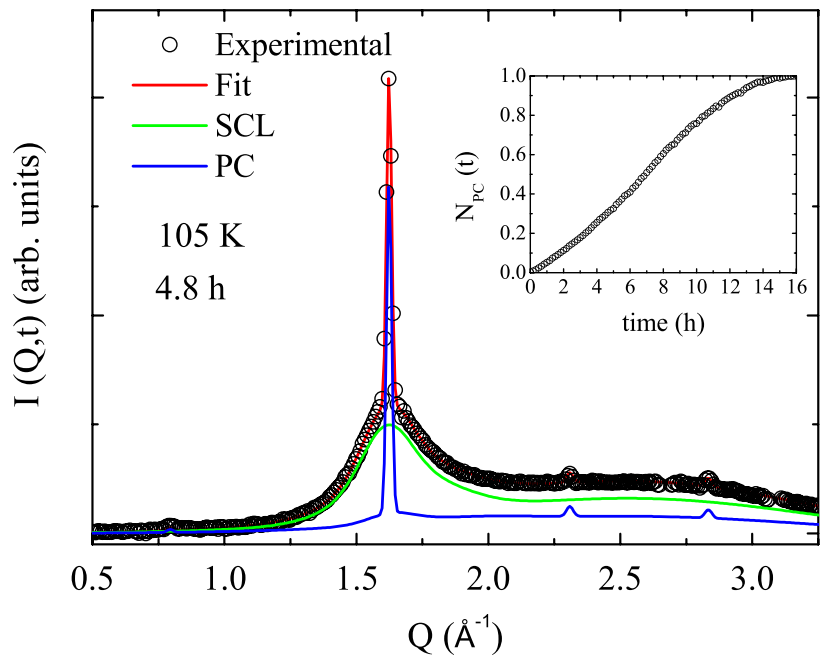

FIG. 2 (color online). Selected neutron diffraction pattern $(4.8 \mathrm{~h})$ for isothermal transformation of SCL ethanol into PC at $105 \mathrm{~K}$. The red continuous line is a fit of the total diffraction pattern to a linear combination of the measured diffraction patterns at the initial (SCL, green line) and final (PC, blue line) times. In the inset we show the time dependence of the $\mathrm{PC}$ fraction.

$[27,28]$. Firstly, our interpretation of the data as a sum of two CD processes did not give satisfactory results. In contrast, a much better description of the data was obtained when a superposition of two HN functions for the SCL and $\mathrm{PC}$, respectively, was employed. In a first approach, the dielectric strength is related to the amount of mobile phase (SCL or PC in our case) while the fraction of PC $\left(N_{\mathrm{PC}}\right)$ is also directly related to the portion of material included in the bcc lattice and can be estimated directly from the ND patterns as explained before. Therefore, a straightforward comparison between the fraction of sample transformed into the PC phase as a function of annealing time calculated through either dielectric spectroscopy or neutron diffraction can be established. Surprisingly, we found that the values of $N_{\mathrm{PC}}$ were noticeably lower (not shown here) when estimated by ND measurements than those calculated by the dielectric strength, especially for intermediate crystallization times. We can tentatively explain this mismatch if we assume that, although a portion of ethanol molecules are relaxing at a slower rate as compared to the fully disorder SCL phase, these are not included in the ordered bcc lattice yet. Given that the values of PC fraction are unequivocally obtained by the diffraction patterns, we proposed a new strategy to describe the dielectric spectra considering the existence of an additional phase, presumably associated with an intermediate step of the partial ordering of SCL ethanol into the rotator phase. In addition to that, the empirical $\mathrm{HN}$ is an unphysical susceptibility function since the zero-frequency limit is not well defined even though it has been widely used to describe dielectric relaxation in a broad frequency window, especially for polymeric systems. Thus, the imaginary part of the complex dielectric permittivity described by a weighted
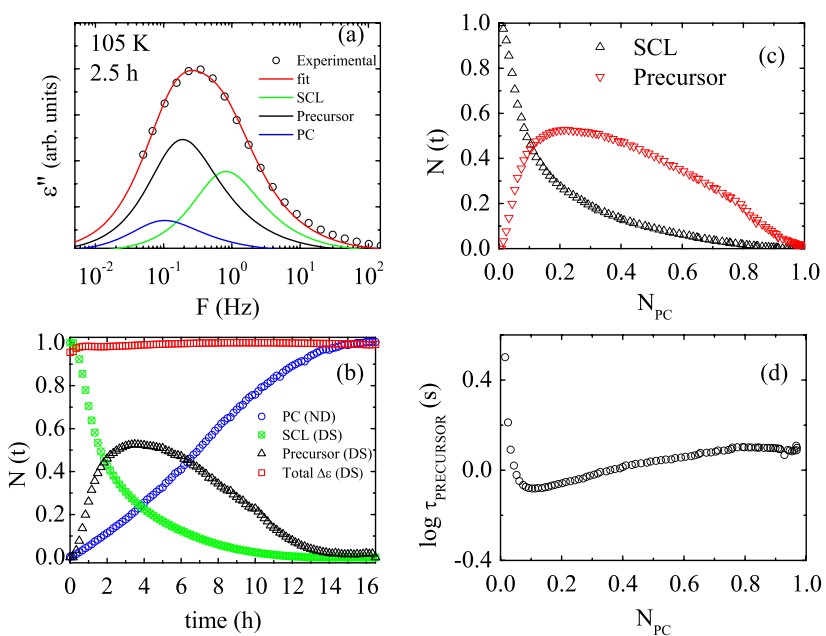

FIG. 3 (color online). (a) Fitting of the dielectric relaxation curve for annealing time of $2.5 \mathrm{~h}$ during transformation of SCL ethanol into PC at $105 \mathrm{~K}$. Green, black, and blue solid lines stand for the SCL, interface, and PC phases, respectively. (b) Time evolution of the fraction associated with the coexisting phases: $\bigcirc$ the PC calculated by ND, $\triangle$ the interface, and $\otimes$ the SCL. Normalized total dielectric strength $(\square)$ is also shown for comparison. (c) Normalized dielectric strength to its initial value for the SCL and interface relaxations of deuterated ethanol as a function of the PC degree of order. (d) Characteristic relaxation time for the main relaxation assigned to the precursor phase as a function of the PC degree of order.

superposition of three CD modes is as follows: $\epsilon^{\prime \prime}(\omega, t)=$ $N_{\mathrm{PC}}(t) \epsilon_{\mathrm{PC}}^{\prime \prime}(\omega)+N_{\mathrm{PRE}}(t) \epsilon_{\mathrm{PRE}}^{\prime \prime}(\omega)+N_{\mathrm{SCL}}(t) \epsilon_{\mathrm{SCL}}^{\prime \prime}(\omega)$, where the subscripts stand for the initial SCL and final PC phases, and also for the precursor (PRE) phase. As stated above, considering the information obtained by ND is correct and in order to minimize the number of adjustable parameters, we performed the fitting of the dielectric spectra using the values of $N_{\text {PC }}$ estimated by the ND measurements. In Fig. 3(a) we show, as an example, the best fit of the dielectric spectrum to the weighted superposition function described above for an intermediate annealing time of $2.5 \mathrm{~h}$. Contributions from the three coexisting phases, modeled as CD functions, are highlighted by different colors and give a robust fitting of the experimental data. As a result of this analysis, Fig. 3(b) shows the time evolution of the normalized dielectric strength of the SCL and intermediate or precursor phase relaxations. The fraction for the PC phase obtained by ND is also included. The fraction of SCL phase drastically decreases for the early stages of the partial ordering process. This period coincides with an abrupt increase of the fraction of the precursor phase. The amount of molecules in the precursor or intermediate phase reaches a maximum of 0.5 at approximately $3 \mathrm{~h}$, showing then a monotonic decrease as expenses of the growth of the final PC phase. We also correlate the fraction of SCL and precursor phases to the degree of (plastic) crystallinity in Fig. 3(c). Here, one can see that, for the early stages, the sample is mostly 
occupied by the starting SCL and precursor phases. In parallel, the characteristic dynamics of the precursor phase becomes slightly faster, showing afterwards a moderate slowing down during the (plastic) crystalline growth phase as indicated in Fig. 3(d). This panel specifically collects the dependence of the precursor phase relaxation time as a function of the fraction of sample transformed into the PC phase. Unlike it was previously reported by Benkhof et al. [5], in our case the presence of the precursor phase prevents the observation of the isosbestic point in the dielectric spectra. They considered that just two phases coexisted during the transition, that is, the pure SCL and PC phases. The discrepancy between the PC fraction estimated by dielectric and neutron measurements shown by our results gives further support to our idea that, previous to the formation of the rotator phase, the growth of an intermediate phase must take place. However, both pictures might be reconciled if different crystallization mechanisms are adopted depending on the sample environment. Here, our deuterated ethanol is placed in a holder made of anodized aluminum and the partial ordering of SCL ethanol seems to be firstly driven by a liquid-liquid phase separation during the early stages and subsequent homogeneous (plastic) crystalline growth. During the initial development of the proposed liquid-liquid phase separation, the relaxation dynamics of the precursor phase undergoes a slight acceleration, probably connected to the breakage or disruption of the hydrogen-bonding network, and straight afterwards, as the precursor phase progressively transforms into the PC phase, it shows a subtle slowing down as indicated in Fig. 3(d). A similar interpretation has been recently reported to explain the cold crystallization of a polymeric system like poly(trimethylene terephthalate) [29]. On the contrary, Benkhof's results are better suited for an heterogeneous nucleation. In this case, they used glass plates covered with gold as electrodes. Presumably the spreading of gold particles, isotropically dispersed along the sample, could act as starting nucleation spots, initiating heterogeneously the transition of SCL ethanol into PC form.

This work has been funded by the Spanish CICYT (MAT2009-07789 and MAT2008-03232). A. S. also acknowledges the Higher Council for Scientific Research (CSIC) for financial support. The authors thank the ILL for beam time and technical assistance during experiments. We also thank the Program MODELICO-CM.

*asanz@iem.cfmac.csic.es

[1] P. Debenedetti, Metastable Liquids: Concepts and Principles (Princeton University Press, Princeton, 1996), Vol. 441, p. 168.

[2] R. A. L. Jones, in Soft Condensed Matter, Oxford Master Series in Condensed Matter Physics Vol. 6 (Oxford University Press, Oxford, 2002).

[3] P. Debenedetti, Nature (London) 441, 168 (2006).
[4] C. Cabrillo, F. Bermejo, M. Jiménez-Ruiz, M. FernándezDíaz, M. Gonzalez, and D. Marero, Phys. Rev. B 64, 064206 (2001).

[5] S. Benkhof, A. Kudlik, T. Blochowicz, and E. Rossler, J. Phys. Condens. Matter 10, 8155 (1998).

[6] M. Descamps, N. T. Correia, P. Derollez, F. Danede, and F. Capet, J. Phys. Chem. B 109, 16092 (2005).

[7] D. L. Price, M. L. Saboungi, and F. J. Bermejo, Rep. Prog. Phys. 66, 407 (2003).

[8] M. Winterlich, H. Zimmermann, and R. Bohmer, J. NonCryst. Solids 307-310, 442 (2002).

[9] A. Criado, M. Jiménez-Ruiz, C. Cabrillo, F. J. Bermejo, R. Fernández-Perea, H.E. Fischer, and F. R. Trouw, Phys. Rev. B 61, 12082 (2000).

[10] L. Granasy, T. Pusztai, T. Borzsonyi, J. A. Warren, and J. F. Douglas, Nature Mater. 3, 645 (2004).

[11] O. Haida, H. Suga, and S. Seki, J. Chem. Thermodyn. 9, 1133 (1977).

[12] A. Srinivasan, F. Bermejo, A. de Andres, J. Dawidowski, J. Zuniga, and A. Criado, Phys. Rev. B 53, 8172 (1996).

[13] F. J. Bermejo, A. Criado, R. Fayos, R. Fernández-Perea, H. E. Fischer, E. Suard, A. Guelylah, and J. Zuniga, Phys. Rev. B 56, 11536 (1997).

[14] R. Fayos, F. Bermejo, J. Dawidowski, H. Fischer, and M. Gonzalez, Phys. Rev. Lett. 77, 3823 (1996).

[15] M. A. Ramos, V. Rodríguez-Mora, and R. J. JiménezRioboo, J. Phys. Condens. Matter 19, 205135 (2007).

[16] M. Jiménez-Ruiz, M. A. González, F. J. Bermejo, M. A. Miller, N. O. Birge, I. Cendoya, and A. Alegría, Phys. Rev. B 59, 9155 (1999).

[17] M. Jiménez-Ruiz, A. Criado, F. J. Bermejo, G. J. Cuello, F. R. Trouw, R. Fernández-Perea, H. Lowen, C. Cabrillo, and H. E. Fischer, Phys. Rev. Lett. 83, 2757 (1999).

[18] R. Brand, P. Lunkenheimer, U. Schneider, and A. Loidl, Phys. Rev. B 62, 8878 (2000).

[19] M. Gonzalez, F. Bermejo, E. Enciso, and C. Cabrillo, Philos. Mag. 84, 1599 (2004).

[20] M. Jiménez-Ruiz, A. Sanz, A. Nogales, and T. Ezquerra, Rev. Sci. Instrum. 76, 043901 (2005).

[21] P. Petong, R. Pottel, and U. Kaatze, J. Phys. Chem. A 103, 6114 (1999).

[22] C. Gainaru, R. Meier, S. Schildmann, C. Lederle, W. Hiller, E. A. Roessler, and R. Boehmer, Phys. Rev. Lett. 105, 258303 (2010).

[23] L. Wang, S. Shahriari, and R. Richert, J. Phys. Chem. B 109, 23255 (2005).

[24] M. A. Ramos, S. Vieira, F. J. Bermejo, J. Dawidowski, H. E. Fischer, H. Schober, M. A. González, C. K. Loong, and D. L. Price, Phys. Rev. Lett. 78, 82 (1997).

[25] A. Sanz, M. Jiménez-Ruiz, A. Nogales, D. Martín y Marero, and T. A. Ezquerra, Phys. Rev. Lett. 93, 015503 (2004).

[26] A. Sanz, A. Nogales, T. A. Ezquerra, M. Soccio, A. Munari, and N. Lotti, Macromolecules 43, 671 (2010).

[27] S. Havriliak and S. Negami, Polymer 8, 161 (1967).

[28] A. Schonhals and F. Kremer, Broad Band Dielectric Spectroscopy (Springer, Berlin, 2002).

[29] W. T. Chuang, W. B. Su, U. S. Jeng, P. D. Hong, C. J. Su, C.H. Su, Y.C. Huang, K.F. Laio, and A.C. Su, Macromolecules 44, 1140 (2011). 